\title{
Correction to: Fast random field generation with $\mathrm{H}$-matrices
}

\author{
Michael Feischl ${ }^{1}$. Frances Y. Kuo ${ }^{2}$ - Ian H. Sloan ${ }^{2}$
}

Published online: 21 March 2019

๑) Springer-Verlag GmbH Germany, part of Springer Nature 2019

\section{Correction to: Numer. Math. (2018) 140:639-676 https://doi.org/10.1007/s00211-018-0974-2}

On page 3 in line -4 , the correct statement is

$\ldots \mu \in(0, \infty], p \in 2 \mathbb{N}$ are parameters.

The corrected version states the parameter range as $p \in 2 \mathbb{N}$ instead of $p \in \mathbb{N}$. The effect is to disallow non-smooth norms such as the $\ell_{1}$-norm for the distance measure. This, however, does not impact the usual definition of the Matérn class of covariance functions using the $\ell_{2}$-norm for all values of $\mu \in(0, \infty]$ (particularly the exponential case for $\mu=1 / 2$ and the Gaussian case for $\mu=\infty)$. Moreover, all the numerical examples and particularly the non-stationary covariance functions on page 642 are still covered by the theory.

This minor change closes the small gap in the proof of Lemma 1 by ensuring the existence of all the derivatives which are shown to be bounded in the sense of asymptotic smoothness (1).

Publisher's Note Springer Nature remains neutral with regard to jurisdictional claims in published maps and institutional affiliations.

The original article can be found online at https://doi.org/10.1007/s00211-018-0974-2.

Michael Feischl

michael.feischl@kit.edu

Frances Y. Kuo

f.kuo@unsw.edu.au

Ian H. Sloan

i.sloan@unsw.edu.au

1 Karlsruhe Institute of Technology, 76131 Karlsruhe, Germany

2 School of Mathematics and Statistics, UNSW Sydney, Sydney, NSW 2052, Australia 